\title{
A NOTE ON THE GEOMETRIC MEANS OF ENTIRE FUNCTIONS OF SEVERAL COMPLEX VARIABLES
}

BY

\section{P. K. KAMTHAN}

ABSTRACT. Let $f\left(z_{1}, \cdots, z_{n}\right)$ be an entire function of $n(\geq 2)$ complex variables. Recently Agarwal [Trans. Amer. Math. Soc. 151 (1970), 651657] has obtained certain results involving geometric mean values of $f$. In this paper we have constructed examples to contradict some of the results of Agarwal and have thereafter given improvements and modifications of his results.

1. Introduction. Let

$$
f\left(z_{1}, z_{2}\right)=\sum_{m, n \geq 0} a_{m n} z_{1}^{m} z_{2}^{n}
$$

be an entire function of two complex variables (we consider the two variables case for the sake of simplicity). Let

$$
M\left(r_{1}, r_{2}\right)=\max _{\left|z_{1}\right| \leq r_{1},\left|z_{2}\right| \leq r_{2}}\left|f\left(z_{1}, z_{2}\right)\right|
$$

$$
G\left(r_{1}, r_{2}\right)=\exp \left\{\frac{1}{(2 \pi)^{2}} \int_{0}^{2 \pi} \int_{0}^{2 \pi} \log \left|f\left(r_{1} e^{i \theta},_{r_{2}} e^{i \theta_{2}}\right)\right| d \theta_{1} d \theta_{2}\right\}
$$

$$
g_{k, \lambda}\left(r_{1}, r_{2}\right)=\exp \left\{\frac{(k+1)(\lambda+1)}{r_{1}^{k+1} r_{2}^{\lambda+1}} \int_{0}^{r} \int_{0}^{r} 2 x_{1}^{k} x_{2}^{\lambda} \log G\left(x_{1}, x_{2}\right) d x_{1} d x_{2}\right\},
$$

where $0<k, \lambda<\infty$, be the geometric means of $f\left(z_{1}, z_{2}\right)$. The term $g_{k, \lambda}\left(r_{1}, r_{2}\right)$ and its various properties were probably first considered as early as in 1962 by the author [2] in terms of an entire function of a single variable. Recently, Agarwal [1] has generalised some of the results in [3] in terms of $G\left(r_{1}, r_{2}\right)$ and $g_{k, \lambda}\left(r_{1}, r_{2}\right)$ when $k=\lambda$, and in addition has also proved the following:

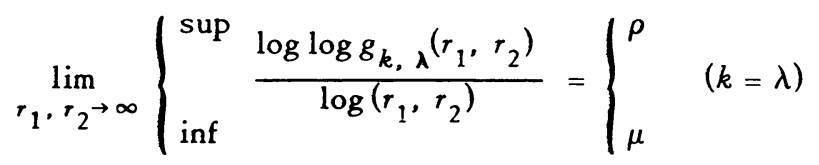

Received by the editors July 15, 1971.

AMS 1969 subject classifications. Primary 3205, 3210; Secondary 3217.

Key words and phrases. Entire function, geometric means, order, Poisson formula for two variables, slowly changing function. 
where $\left.{ }^{1}\right)$

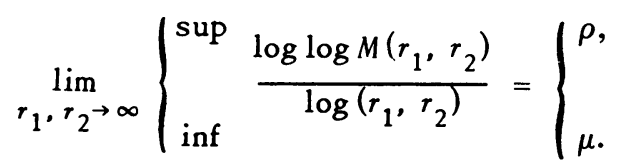

Apart from giving certain growth results involving $G\left(r_{1}, r_{2}\right)$ and $g_{k, \lambda}\left(r_{1}, r_{2}\right)$, our chief aim is to present an example which violates (1.3)-an improvement of which (i.e. a correct version of (1.3)) is given in $\$ 2$ that follows now.

2. A counterexample for (1.3) and its improvement. Let $f\left(z_{1}, z_{2}\right)=e^{z_{1} z_{2}}$. Then $M\left(r_{1}, r_{2}\right)=e^{r_{1} r_{2}}$. Therefore $\rho=\mu=1$. Now

$$
\begin{gathered}
\log \left|f\left(r_{1} e^{i \theta_{1}}, r_{2} e^{i \theta_{2}}\right)\right|=r_{1} r_{2} \cos \theta_{1} \cos \theta_{2}-r_{1} r_{2} \sin \theta_{1} \sin \theta_{2} \\
\Rightarrow G\left(r_{1}, r_{2}\right)=1, \text { for all } r_{1}, r_{2}>0 .
\end{gathered}
$$

Hence $\log g_{k, k}\left(r_{1}, r_{2}\right)=0$ for all $r_{1}, r_{2}>0$. Thus if (1.3) is true then $\rho=\mu=-\infty$ which is absurd. We may lead to a similar discussion if $f\left(z_{1}, z_{2}\right)=\exp \left(z_{1}+z_{2}\right)$ and the details are left to the reader. I may point out that the main fault in establishing (1.3) is the following inequality (see line 4 from above, p. 653 of [1]) which Agarwal has proved:

$$
\log g_{k, k}\left(\alpha r_{1}, \alpha r_{2}\right) \geq\{(\alpha-1) /(\alpha+1)\}^{2}\left\{1-1 / \alpha^{k+1}\right\}^{2} \log M\left(r_{1} / \alpha, r_{2} / \alpha\right), \quad \alpha>1,
$$

and which is also incorrect in view of the above example.

To offer an improvement of (1.3), let us define first

$$
G^{+}\left(r_{1}, r_{2}\right)=\exp \left\{\frac{1}{(2 \pi)^{2}} \int_{0}^{2 \pi} \int_{0}^{2 \pi} \log { }^{+}\left|f\left(r_{1} e^{i \theta_{1}}, r_{2} e^{i \theta_{2}}\right)\right| d \theta_{1} d \theta_{2}\right\}
$$

where

$$
\log ^{+}|f|=\max (\log |f|, 0)
$$

also let

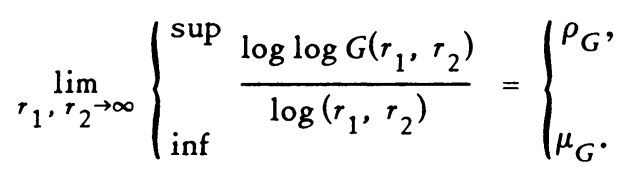

We have then the following result:

Theorem 2.1. If $f\left(z_{1}, z_{2}\right)$ is an entire function, then for $R_{1}>r_{1}, R_{2}>r_{2}$,

$$
\log G^{+}\left(r_{1}, r_{2}\right) \leq \log ^{+} M\left(r_{1}, r_{2}\right) \leq \frac{R_{1}+r_{1}}{R_{2}+r_{2}} \frac{R_{2}+r_{2}}{R_{2}-r_{2}} \log G^{+}\left(R_{1}, R_{2}\right),
$$

(1) Agarwal's claim that $\rho$ and $\mu$ are nonintegral is irrelevant as far as the proof of (1.3) goes. 
and

$$
\lim _{r_{1}, r_{2} \rightarrow \infty}\left\{\begin{array}{l}
\text { sup } \\
\text { inf }
\end{array}\right.
$$

for any $k, \lambda$ such that $0<k, \lambda<\infty$.

Proof. (2.1) immediately follows from Poisson's inequality in two variables. For (2.2), we observe

$$
\begin{aligned}
\log g_{k, \lambda}\left(r_{1}, r_{2}\right) & \leq\left\{\frac{(k+1)(\lambda+1)}{r_{1}^{k+1} r_{2}^{\lambda+1}} \int_{0}^{r} 1 \int_{0}^{r} x_{1}^{k} x_{2}^{\lambda} d x_{1} d x_{2}\right\} \log G\left(r_{1}, r_{2}\right) \\
& =\log G\left(r_{2}, r_{2}\right) .
\end{aligned}
$$

Moreover

$$
\begin{aligned}
\log g_{k, \lambda}\left(R_{1}, R_{2}\right) \geq & \frac{(k+1)(\lambda+1)}{R_{1}^{k+1} R_{2}^{\lambda+1}} \int_{r_{1}}^{R_{2}} \int_{r_{2}}^{R_{2}} x_{1}^{k} x_{2}^{\lambda} \log G\left(x_{1}, x_{2}\right) d x_{1} d x_{2} \\
& \geq \frac{\left(R_{1}^{k+1}-r_{1}^{k+1}\right)\left(R_{2}^{\lambda+1}-r_{2}^{\lambda+1}\right)}{R_{1}^{k+1} R_{2}^{\lambda+1}} \log G\left(r_{1}, r_{2}\right) .
\end{aligned}
$$

Hence, putting $R_{1}=\alpha r_{1}, R_{2}=\beta r_{2} ; \alpha, \beta>1$,

$$
\log g_{k, \lambda}\left(\alpha r_{1}, \beta r_{2}\right) \geq \frac{\left(\alpha^{k+1}-1\right)\left(\beta^{\lambda+1}-1\right)}{\alpha^{k+1} \beta^{\lambda+1}} \log G\left(r_{1}, r_{2}\right) .
$$

The inequalities (2.3) and (2.4) result in (2.2).

In this section $w>$ offer improvements of Theorem 2, (3.3) and Theorem 3(ii) and (iii) of Agarwal [1].

$$
\begin{aligned}
& \lim _{r_{1}, r_{2} \rightarrow \infty} \int_{\text {inf }}^{\sup } \frac{\log g_{k, \lambda}\left(r_{1}, r_{2}\right)}{\left(r_{1} r_{2}\right)^{\rho} G_{\phi}\left(r_{1}, r_{2}\right)}=\left\{_{q}^{p} \quad(0<q \leq p<\infty),\right.
\end{aligned}
$$

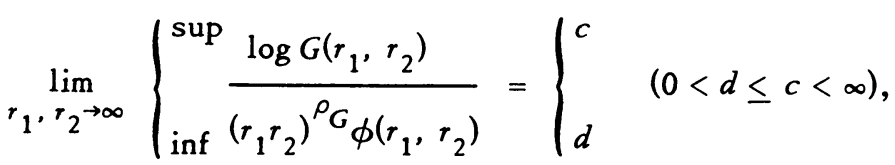

where $\phi\left(r_{1}, r_{2}\right)$ is as mentioned by Agarwal. Then we have

Theorem 3.1. If $f\left(z_{1}, z_{2}\right)$ is an entire function baving finite nonzero value $\rho_{G}$, i.e. $0<\rho_{G}<\infty$, then

$$
\begin{aligned}
d(k+1)(\lambda+1) /\left\{\left(k+\rho_{G}+1\right)\left(\lambda+\rho_{G}+1\right)\right\} & \leq q \leq p \\
& \leq c(k+1)(\lambda+1) /\left\{\left(k+\rho_{G}+1\right)\left(\lambda+\rho_{G}+1\right)\right\} .
\end{aligned}
$$


Proof. The proof is sketched as follows: Let $0<\alpha, \beta<1,0<r_{1}^{0}<r_{1}$, $0<r_{2}^{0}<r_{2}$. Then

$$
\begin{aligned}
& \log g_{k, \lambda}\left(r_{1}+\alpha r_{1}, r_{2}+\beta r_{2}\right) \\
& <\frac{A}{r_{1}^{k+1} r_{2}^{\lambda+1}} \\
& +\frac{1}{(\alpha+1)^{k+1}(\beta+1)^{\lambda+1}}\left\{( \frac { r _ { 1 } ^ { 0 } } { r _ { 1 } } ) ^ { k + 1 } \left[\left\{1-\left(\frac{\beta r_{2}^{0}}{r_{2}}\right)^{\lambda+1}\right\} \log G\left(r_{1}^{0}, r_{2}\right)\right.\right. \\
& \left.+\left\{(1+\beta)^{\lambda+1}-1\right\} \log G\left(r_{1}^{0}, r_{2}+\beta r_{2}\right)\right] \\
& +\left(\frac{r_{2}^{0}}{r_{2}}\right)^{\lambda+1}\left[\left\{1-\left(\frac{\alpha r_{1}^{0}}{r_{1}}\right)^{k+1}\right\} \log G\left(r_{1}, r_{2}^{0}\right)\right. \\
& \left.+\left\{(1+\alpha)^{k+1}-1\right\} \log G\left(r_{1}+\alpha r_{1}, r_{2}^{0}\right)\right] \\
& +\frac{(c+\epsilon)(k+1)(\lambda+1)}{r_{1}^{k+1} r_{2}^{\lambda+1}} \int_{r_{1}^{0}}^{r} \int_{r_{2}^{0}}^{r_{2}} x_{1}^{\rho_{G}+k} x_{2}^{\rho_{G}+\lambda} \phi\left(x_{1}, x_{2}\right) d x_{1} d x_{2} \\
& +\frac{(k+1)\left((1+\beta)^{\lambda+1}-1\right)}{r_{1}^{k+1}} \int_{r_{1}^{0}}^{r_{1}} x_{1}^{k} \log G\left(x_{1}, r_{2}+\beta r_{2}\right) d x_{1} \\
& +\frac{(\lambda+1)\left((1+\alpha)^{k+1}-1\right)}{r_{2}^{\lambda+1}} \int_{r_{2}^{0}}^{r_{2}} x_{2}^{\lambda} \log G\left(r_{1}+\alpha r_{1}, x_{2}\right) d x_{2} \\
& \left.\left.+\left\{(1+\alpha)^{k+1}-1\right\}\{1+\beta)^{\lambda+1}-1\right\} \log G\left(r_{1}+\alpha r_{1}, r_{2}+\beta r_{2}\right)\right\} \text {. }
\end{aligned}
$$

Next, observe that the seventh, eighth, and ninth lines of the foregoing inequality are respectively equal at most to the following estimates:

$$
\frac{(c+\epsilon)(k+1)(\lambda+1)}{(1+\alpha)^{k+1}(1+\beta)^{\lambda+1}\left(\rho_{G}+k+1\right)\left(\rho_{G}+\lambda+1\right)} \phi\left(r_{1}, r_{2}\right)\left(r_{1} r_{2}\right)^{\rho_{G}},
$$

$$
\frac{(k+1)(1+\beta)^{\rho} G\left((1+\beta)^{\lambda+1}-1\right)}{(1+\alpha)^{k+1}(1+\beta)^{\lambda+1}\left(\rho_{G}+k+1\right)} \phi\left(r_{1}, r_{2}+\beta r_{2}\right)\left(r_{1} r_{2}\right)^{\rho} G,
$$

$$
\frac{(\lambda+1)(1+\alpha)^{\rho} G_{\left((1+\alpha)^{k+1}-1\right)}}{(1+\alpha)^{k+1}(1+\beta)^{\lambda+1}\left(\rho_{G}+\lambda+1\right)} \phi\left(r_{1}+\alpha r_{1}, r_{2}\right)\left(r_{1} r_{2}\right)^{\rho} \text {. }
$$


Making use of these estimates in the corresponding terms of the above inequality, then dividing the complete expression by

$$
\left.\left(r_{1}+\alpha r_{1}\right)^{\rho} G_{\left(r_{2}\right.}+\beta r_{2}\right)^{\rho} G_{\phi}\left(r_{1}+\alpha r_{1}, r_{2}+\beta r_{2}\right)
$$

and finally proceeding to the limit as $r_{1}, r_{2} \rightarrow \infty$, one gets the following: namely,

$$
\begin{aligned}
& p \leq \frac{c}{(1+\alpha)^{k+1}(1+\beta)^{\lambda+1}}\left\{\frac{(k+1)(1+\beta)^{\rho}{ }^{\rho}\left((1+\beta)^{\lambda+1}-1\right)}{\rho_{G}+k+1}\right. \\
& -\frac{(\lambda+1)(1+\alpha)^{\rho}{ }^{\rho}\left((1+\alpha)^{k+1}-1\right)}{\rho_{G}+\lambda+1} \\
& \left.+\frac{(k+1)(\lambda+1)}{\left(\rho_{G}+k+1\right)\left(\rho_{G}+\lambda+1\right)}\right\} \text {. }
\end{aligned}
$$

But, $\alpha, \beta$ are arbitrary and so making $\alpha, \beta \rightarrow 0$, we find that the right-hand inequality in (3.3) is established.

Next, we have from (1.2) for all sufficiently large values of $r_{1}$ and $r_{2}$, $\log g_{k, \lambda}\left(r_{1}+\alpha r_{1}, r_{2}+\beta r_{2}\right)$

$$
\begin{aligned}
& >\frac{(d-\epsilon)(k+1)(\lambda+1)}{r_{1}^{k+1} r_{2}^{\lambda+1}(1+\alpha)^{k+1}(1+\beta)^{\lambda+1}} \int_{r_{1}^{0}}^{r_{1}} \int_{r_{2}^{0}}^{r_{2}} x_{1}^{\rho_{G}+k} x_{2}^{\rho_{G}+\lambda} \phi\left(x_{1}, x_{2}\right) d x_{1} d x_{2} \\
& +\frac{1}{(1+\alpha)^{k+1}(1+\beta)^{\lambda+1}}\left\{\frac{(k+1)\left((1+\beta)^{\lambda+1}-1\right)}{r_{1}^{k+1}} \int_{r_{1}^{0}}^{r_{1}} x_{1}^{k} \log G\left(x_{1}, r_{2}\right) d x_{1}\right. \\
& +\frac{(\lambda+1)\left((1+\alpha)^{k+1}-1\right)}{r_{2}^{\lambda+1}} \int_{r_{2}}^{r_{2}} x_{2}^{\lambda} \log G\left(r_{1}, x_{2}\right) d x_{2} \\
& \left.+\left\{(1+\alpha)^{k+1}-1\right\}\left\{(1+\beta)^{\lambda+1}-1\right\} \log G\left(r_{1}, r_{2}\right)\right\} \text {. }
\end{aligned}
$$

Observe that

$$
\begin{aligned}
& \log G\left(x_{1}, r_{2}\right)>(d-\epsilon)\left(x_{1} r_{2}\right)^{\rho} G_{\phi}\left(x_{1}, r_{2}\right), \text { for } x_{1}>r_{1}^{0}, \\
& \log G\left(r_{1}, x_{2}\right)>(d-\epsilon)\left(r_{1} x_{2}\right)^{\rho} G_{\phi}\left(r_{1}, x_{2}\right), \text { for } x_{2}>r_{2}^{0}, \\
& \log G\left(r_{1}, r_{2}\right)>(d-\epsilon)\left(r_{1} r_{2}\right){ }^{\rho} G \phi\left(r_{1}, r_{2}\right), \text { for } r_{1}>r_{1}^{0}, r_{2}>r_{2}^{0} .
\end{aligned}
$$


Hence

$$
\begin{aligned}
(1+\alpha)^{\rho_{G}+k+1}(1+\beta)^{\rho_{G}+\lambda+1} q \geq & \frac{(k+1)(\lambda+1) d}{\left(\rho_{G}+k+1\right)\left(\rho_{G}+\lambda+1\right)}+\frac{(k+1)\left((1+\beta)^{\lambda+1}-1\right) d}{k+\rho_{G}+1} \\
& +\frac{(\lambda+1)\left((1+\alpha)^{k+1}-1\right) d}{\lambda+\rho_{G}+1} \\
& +\left\{(1+\alpha)^{k+1}-1\right\}\left\{(1+\beta)^{\lambda+1}-1\right\} d,
\end{aligned}
$$

and making now $\alpha, \beta \rightarrow 0$, the left-hand inequality in (3.3) is obtained.

Invoking Theorem 2 and the technique of its proof as envisaged in [3] together with the method adopted in the proof of the above theorem, one may now easily prove the following:

Theorem 3.2. If $f\left(z_{1}, z_{2}\right)$ is an entire function, such that $c=d$, then $p=q=(k+1)(\lambda+1) c /\left\{k+\rho_{G}+1\right\}\left\{\lambda+\rho_{G}+1\right\}$, and

$$
\lim _{r_{1}, r_{2} \rightarrow \infty} \frac{\log g_{k, \lambda}\left(r_{1}, r_{2}\right)}{\log G\left(r_{1}, r_{2}\right)}=\frac{(k+1)(\lambda+1)}{\left(k+\rho_{G}+1\right)\left(\lambda+\rho_{G}+1\right)} .
$$

Remark. The author is of the view that the results (3.4) and (3.5) of Agarwal may not be generalised in terms of $\log g_{k, \lambda}\left(r_{1}, r_{2}\right)$ when $k \neq \lambda$ and are arbitrary. Attempts towards these generalisations involve enormous calculations without yielding any solid solution.

\section{REFERENCES}

1. A. K. Agarwal, On the geometric means of entire functions of several complex variables, Trans. Amer. Math. Soc. 151 (1970), 651-657.

2. P. K. Kamthan, On the mean values of an entire function, Math. Student 32 (1964), 101-109. MR $32 \# 4270$.

3. P. K. Kamthan and P. K. Jain, The geometric means of an entire function, Ann. Polon. Math. 21 (1968/69), 247-255. MR 39 \#4396. U. P., INDIA

DEPARTMENT OF MATHEMATICS, INDIAN INSTITUTE OF TECHNOLOGY, KANPUR-16, 